\title{
The NAOMI Adaptive Optics System for the 4.2m William Herschel Telescope ${ }^{*}$
}

\author{
Richard M. Myers ${ }^{\mathrm{a}}$, Andrew J. Longmore ${ }^{\mathrm{b}}$, Christopher R. Benn ${ }^{\mathrm{c}}$, David F. Buscher ${ }^{\mathrm{d}}$, Paul Clark ${ }^{\mathrm{a}}$, \\ Nigel A. Dipper ${ }^{\mathrm{a}}$, Nathan Doble ${ }^{\mathrm{e}}$, A. Peter Doel ${ }^{\mathrm{f}}$, Colin N. Dunlop $^{\mathrm{a}}$, Xiaofeng Gao ${ }^{\mathrm{b}}$, \\ Thomas Gregory ${ }^{\mathrm{c}}$, Ronald A. Humphreys ${ }^{\mathrm{a}}$, Derek Ives ${ }^{\mathrm{b}}$, Roy Østensen ${ }^{\mathrm{c}}$, P. Tully Peacocke ${ }^{\mathrm{b}}$, \\ René G.M. Rutten ${ }^{\mathrm{c}}$, Christopher J. Tierney ${ }^{\mathrm{b}}$, Andrew J.A. Vick ${ }^{\mathrm{b}}$, Martyn R. Wells ${ }^{\mathrm{b}}$, \\ Richard W. Wilson ${ }^{\mathrm{a}}$, Susan P. Worswick ${ }^{\mathrm{g}}$, Andrew Zadrozny ${ }^{\mathrm{a}}$
${ }^{a}$ University of Durham, UK; ${ }^{b}$ UK Astronomy Technology Centre, Royal Obervatory Edinburgh;
${ }^{\mathrm{c}}$ Isaac Newton Group of Telescopes, Spain; ${ }^{\mathrm{d}}$ University of Cambridge, UK;
${ }^{\mathrm{e}}$ University of Rochester, NY; ${ }^{\mathrm{f}}$ University College London, UK; ${ }^{\mathrm{g}}$ Observatory Optics, UK

\begin{abstract}
NAOMI (Nasmyth Adaptive Optics for Multi-purpose Instrumentation) is a recently completed and commissioned astronomical facility on the $4.2 \mathrm{~m}$ William Herschel Telescope. The system is designed to work initially with Natural Guide Stars and also to be upgradeable for use with a single laser guide star. It has been designed to work with both near infrared and optical instrumentation (both imagers and spectrographs). The system uses a linearised segmented adaptive mirror and dual-CCD Shack-Hartmann wavefront sensor together with a multiple-DSP real-time processing system. Control system parameters can be updated on-the-fly by monitoring processes and the system can self-optimize its base optical figure to compensate for the optical characteristics of attached scientific instrumentation. The scientific motivation, consequent specification and implementation of NAOMI are described, together with example performance data and information on future upgrades and instrumentation.
\end{abstract}

Keywords: Adaptive Optics, Natural Guide Stars, Segmented Mirror

\section{INTRODUCTION}

The William Herschel Telescope (WHT) is a $4.2 \mathrm{~m}$ alt-azimuth mounted telescope equipped with Nasmyth platforms. One of the Nasmyth platforms is configured with an optical table in a light-tight enclosure: the Ground-Based High Resolution Imaging Laboratory (GHRIL). It is the GHRIL station at which the NAOMI AO system and its instrumentation are currently deployed.

The novel components of the NAOMI adaptive mirror and control system were prototyped with the University of Durham ELECTRA (ELEment Cophasing and TRAcking) Adaptive Optics system ${ }^{1}$. Like NAOMI, ELECTRA can also be deployed at the WHT/GHRIL, albeit not in the form of a facility or "common-user" instrument that may be operated and maintained by the observatory.

\section{SCIENTIFIC REQUIREMENTS}

The following bullet list gives an abridged version of the NAOMI requirements on which real time system specifications, overall layout, the wavefront sensing spatial order and other key design choices were based. Rather than attempt to specify a performance under all acceptable conditions, the approach was to define only the end points of the

\footnotetext{
* The William Herschel Telescope is operated on the island of La Palma by the Isaac Newton Group of Telescopes in the Spanish Observatorio del Roque de los Muchachos of the Instituto de Astrofísica de Canarias.
} 
requirements: the Strehl ratio and sky coverage the system must achieve in optimal conditions and in the worst conditions under which it must still provide some minimal level of AO correction.

- The AO system shall be capable of delivering an output Strehl ratio of at least $65 \%$ on-axis at a wavelength of $2.2 \mu \mathrm{m}$ with guide stars of $\mathrm{R}$ magnitude 8 or brighter when the visible $(0.55 \mu \mathrm{m}$ wavelength $)$ atmospheric coherence length $\mathrm{r}_{\mathrm{O}}$ is $20 \mathrm{~cm}$ or larger.

- At the same correction wavelength and $r_{0}$ as above, the system should aim to achieve at least $25 \%$ Strehl ratio at the centre of the science field with $25 \%$ sky coverage. Any failure to meet these specifications shall be due to atmospheric limitations alone.

- The system shall give a net gain in the imaging signal-to-noise ratio of a point source at wavelengths ranging from $0.82 \mu \mathrm{m}$ to $4.1 \mu \mathrm{m}$ in atmospheric turbulence conditions as poor as $r_{0}=8 \mathrm{~cm}$ and with stars at least as faint as visual magnitude 14 .

- The system total IR emissivity, including the telescope, should be minimised as far as practicable and shall be less than $45 \%$ at $2.2 \mu \mathrm{m}$.

- The system should be designed so that it will work, or can be easily modified to work, with a laser beacon guide star.

Along with other subsidiary science performance and operational requirements ${ }^{2}$, these top-level specifications were used to allocate a set of subsystem error budgets, which in turn led to the specification of the subsystems described below.

\section{PRINCIPAL SUBSYSTEMS}

The three main physical sub-assemblies of NAOMI are the Opto-Mechanical Chassis (OMC), the Wavefront Sensor module (WFS), and the Nasmyth Calibration Unit (NCU). The OMC is essentially a precision baseplate, which other components are either mounted on or aligned to.

Fig.1: a block diagram of NAOMI, which shows the relationship between these main sub-assemblies and the control system. The main optical path is shown from the Nasmyth derotator through to the science ports and the WFS CCD.

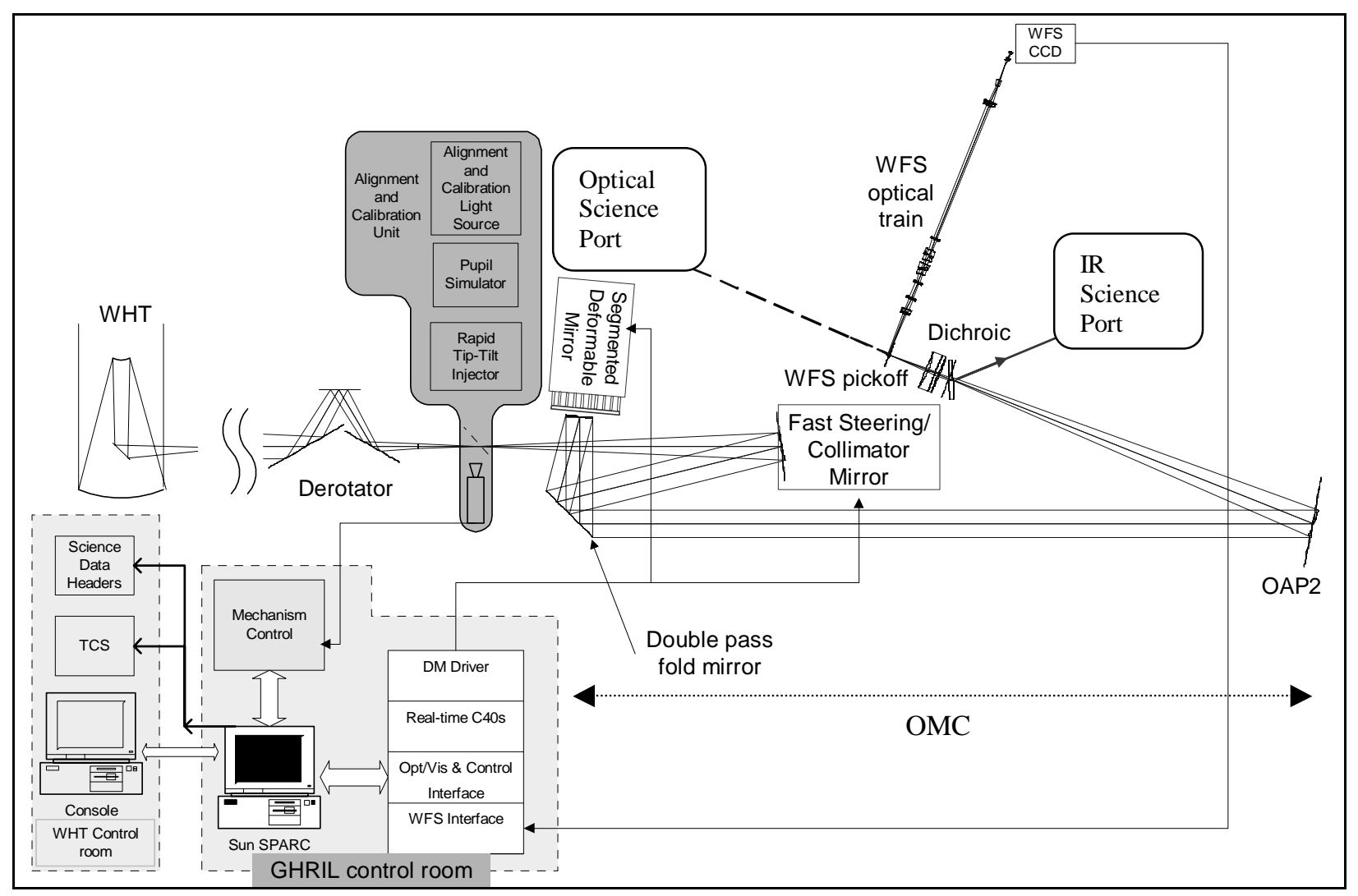


Light is injected from the NCU into this optical path using a removable beamsplitter just before the f/11 Nasmyth focus. The OMC reimages the 2.9 arcmin field of view at the WFS pickoff and science ports; it also contains the fast steering mirror and deformable mirror which correct the distorted wavefront. Shack-Hartmann WFS optics re-image the deformable mirror onto the lenslet array, and then re-image the array of spots produced by the lenslet array onto the WFS CCD. Images from this WFS CCD are analysed by the control system to produce the signals required to drive the fast steering mirror and deformable mirror.

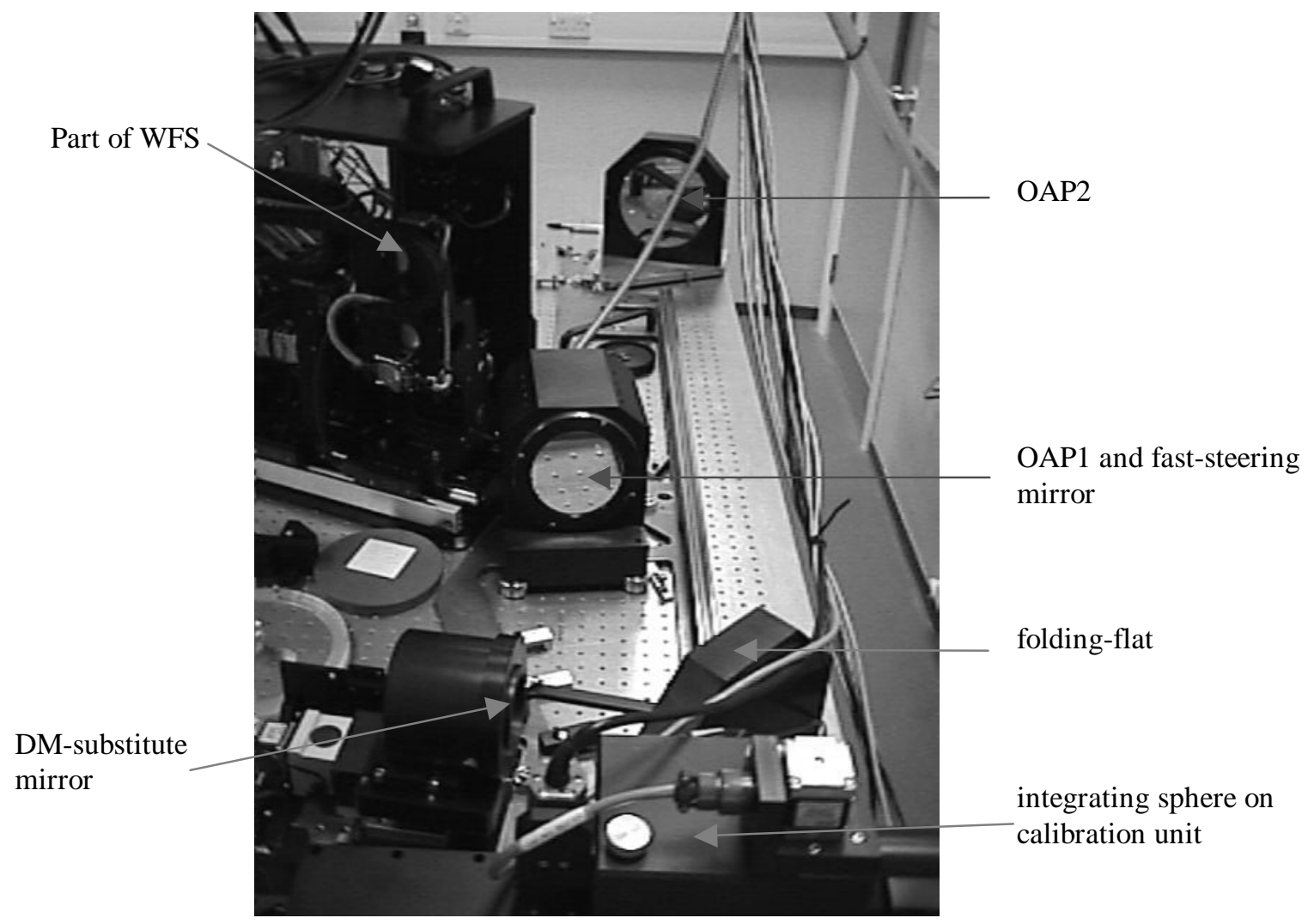

Fig: 2: An image showing NAOMI during laboratory integration. The view is from above the nominal position of the Nasmyth focus, across the top of the NCU and along the OMC. The WFS is on the left and a flat mirror is temporarily replacing the deformable mirror.

The three sub-assemblies are described in turn, followed by descriptions of the Fast Steering Mirror (FSM), Deformable Mirror (DM), real-time control system and supervisory software. Together these constitute the principal subsystems.

\section{THE OPTO-MECHANICAL CHASSIS (OMC).}

This module relays at a magnification of $\sim 1.5$ the whole of the 2.9 arcmin unvignetted field of view available at the f/11 Nasmyth focus via a FSM and a DM to two foci (spectrally split by a dichroic mirror). These foci are referred to as the infrared and the optical science ports. Because the system design is optimised for IR operation, the science camera is placed on the system axis. In operation the movement of the pupil in the IR arm is kept very small. The guide star beam goes through the system, generally off-axis, to a pick-off mechanism that feeds the wavefront sensor.

Collimation and focusing within the OMC is accomplished using two matched off-axis paraboloids. The OMC has been designed for rapid removal from and replacement on the Nasmyth optical bench, and the OAPs and folding flat may be removed, replaced and re-aligned in a few minutes. By 2003, this feature will be used much less as the other Nasmyth focus become available and a permanent adaptive optics station is constructed for NAOMI and the OASIS integral field spectrograph (http://www-obs.univ-lyon1.fr/tiger/home_oasis.html). 


\section{THE WAVEFRONT SENSOR (WFS).}

The WFS measures the wavefront distortions introduced by the atmosphere and telescope, together with the corrections introduced by the FSM and DM. This module picks up the optical light from a guide star after the IR light has been selectively reflected to the IR focus but before it reaches the optical science port. The guide star light is acquired by moving a small pick-off 'probe' mirror in a vertical plane close to (but not at) the output focus of the OMC. An optical fold mirror arrangement converts the effects of $\mathrm{X}$ and $\mathrm{Y}$ motions of the probe into a single focus motion along the optical axis of the WFS. This approach avoids moving the entire WFS. To achieve this the various components of the WFS are mounted on three carriages that can move independently along a single rail. The field available to the probe is unvignetted within the central 2 arcmin of the 2.9 arcmin diameter field passed by the Nasmyth infrared de-rotator.

The WFS uses a Shack-Hartmann configuration in which the input pupil is divided into subapertures by an array of small lenses (lenslets) on a square grid. Each lens focuses the light from the guide star onto a separate region of a detector array, e.g. into a pre-defined 4 x 4 pixel area with a 4 pixel "guard band" between any two such areas. The shift of the focal spot within this area is then determined by one of the processors in the Real-Time Control System (RTCS). The WFS usually operates with 7.3 subapertures across the WHT pupil. In fact, several lenslet arrays are available to deal with changing atmospheric conditions and the brightness of available natural guide stars. The lenslet arrays are mounted on an interchange wheel and may be selected automatically. Two lenslet arrays are available with the 7.3 subaperture sampling described above: one with a longer focal length for good seeing (better than $\sim 0.8$ arcsec at $500 \mathrm{~nm}$ ) and one with half the magnification for poorer seeing. Another lenslet array with half the spatial sampling is available at the higher magnification setting. This is used for fainter guide stars because of the larger collecting area of its subapertures. There is of course a performance penalty from the reduced spatial sampling. This feature is being commissioned on-sky at present and initial results are promising. A further position in the lenslet wheel contains a simple doublet for fast tip-tilt only operation with a very faint guide star (or to use as the NGS sensor in a future LGS configuration). Still other positions in the lenslet wheel contain aids for system alignment and calibration.

The WFS module contains an atmospheric dispersion corrector to allow it to work at zenith angles as large as 60 degrees without undue degradation due to atmospheric dispersion. It also contains a selection of spectral filters mounted in an automated wheel. These assist with the rejection of contaminating background under some conditions.

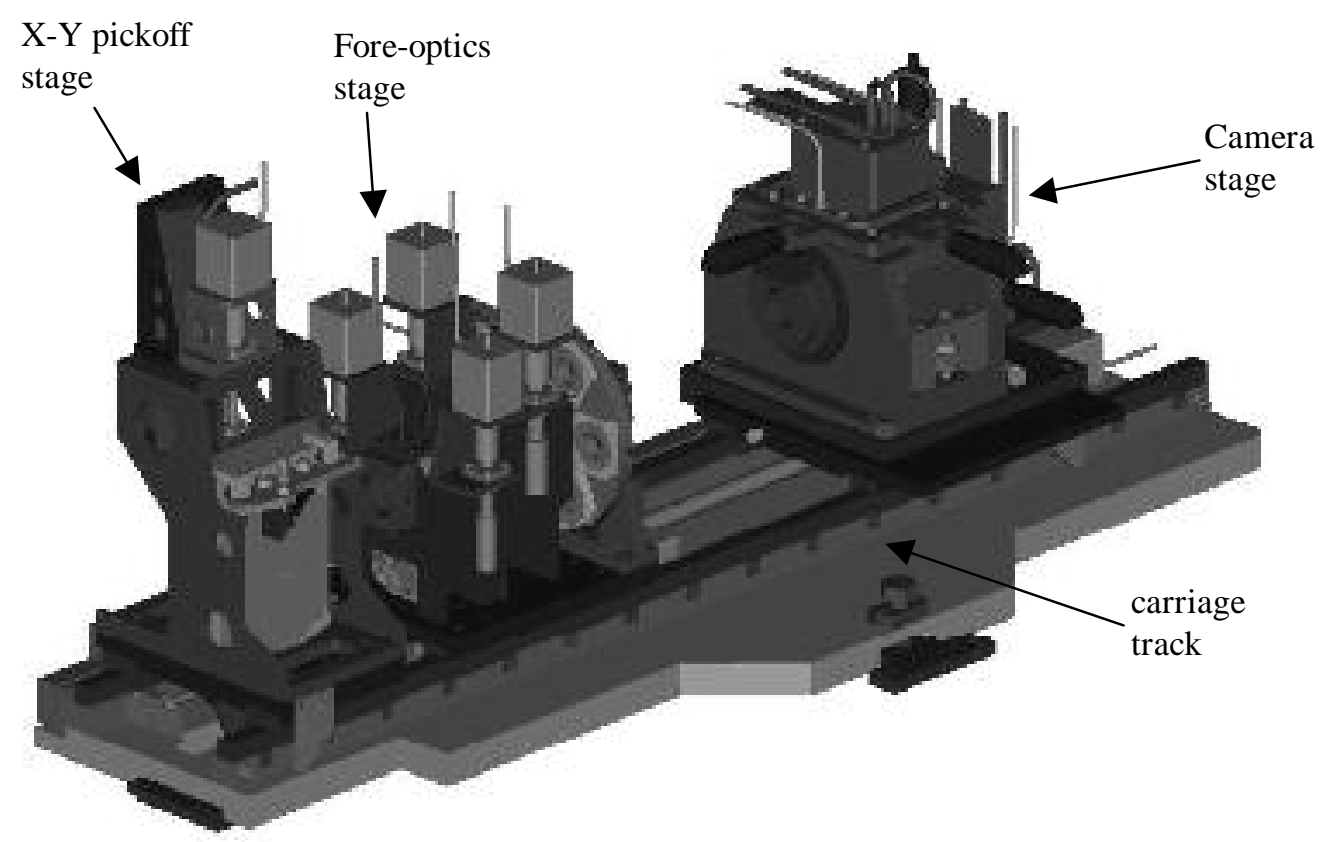

Fig. 3: A 3D CAD model of the WFS module showing the 2D guide star pickoff stage, the fore-optics stage and the camera stage. The fore-optics stage carries the atmospheric dispersion corrector, filter wheel and lenslet wheel. The three stages move independently on the track on which they are mounted. 
A feature of the NAOMI WFS is that it has the capability to use either one or both of two CCDs, after a similar concept used in the SWAT AO system ${ }^{3}$. Single CCD operation is as described above. In two-CCD mode, after the CCD camera optics, the light from the whole wavefront is apportioned equally between the two CCDs by a 50/50 beam-splitter. Pixel data are then binned as required along rows or columns to provide X-direction centroid data from one CCD and Ydirection centroid data from the other. In principle this mode is used when the highest possible closed loop bandwidth is required of the combined WFS / RTCS but it has not yet been commissioned.

The WFS detector system implemented in NAOMI consists of two CCD camera systems, a VME control system and a signal-processing array of Texas Instruments TMS320C44 Digital Signal Processors (DSPs). The digitised image data are fed from the CCD cameras directly to the Real Time Control system DSPs, by-passing the VME bus in the WFS VME control System. The Real Time Control System then performs the image centroiding calculations. The two CCD cameras in the system can be operated in a master and slave mode where one camera is designated the master and the other the slave. In this mode they read out together synchronously with the master supplying the clock and control signals to both cameras. They can also be read out in a single camera mode where either or both of the master and the slave are read out but they are not synchronised. The master/slave mode was implemented to increase system data throughput by the use of horizontal and vertical on-chip binning. The CCD heads are mounted at ninety degrees to each other on the NAOMI carriages.

The CCDs are themselves also electronically divided into subapertures (windows) in which some pixels are skipped over and dumped and others are read out. This again increases system throughput. The CCDs are also binned in different directions for the Master and Slave Controllers. The pattern of skipping and optional binning is adjusted according to which of the lenslet array geometries is in use. The NAOMI WFS camera has two EEV CCD39-01 Dualin-line Package with integral 2-stage Peltier cooler. The CCD39-01 is back illuminated with $80 \times 80$ (plus 4 underscan in each row) pixels, each pixel being $24 \mu \mathrm{m}$ square. The pixels are organized in four quadrants of $40 \times 40$ pixels. Each quadrant has an independent frame-transfer buffer and readout port.

\section{THE NASMYTH CALIBRATION UNIT (NCU).}

This module provides a number of on-axis and off-axis point sources which can be used to calibrate the NAOMI optics. It provides a flat-field source for the science instrument used with NAOMI and a tip-tilt injection facility to test the performance and general 'health' of the adaptive optics components RTCS. It also provides a deployable camera for use in field acquisition.

\section{THE FAST STEERING MIRROR (FSM).}

The fast steering mirror module was constructed by Carl Zeiss (Jena) and is specified to carry the collimating off-axis paraboloid and to reject the measured atmospheric tip-tilt power spectrum. It is a counter-weighted design with internal position feedback. It can produce a beam deflection of 5 arcseconds (on the sky) at frequencies up to $20 \mathrm{~Hz}$ and can produce smaller deflections at higher frequencies.

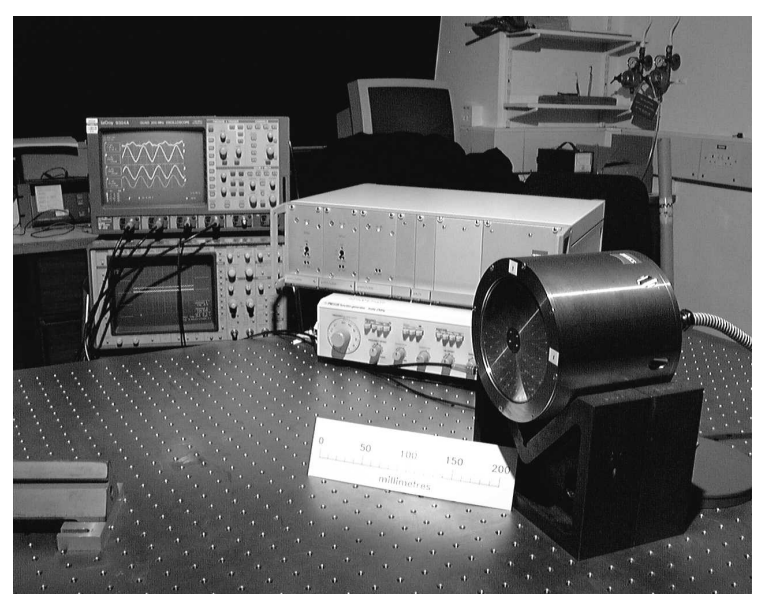

Fig. 4: The Fast steering Mirror drive (foreground right) with its optic dismounted. The drive electronics is in the horizontal rack immediately to the rear. 


\section{THE DEFORMABLE MIRROR (DM).}

The deformable-mirror subsystem corrects for the higher-order, i.e. tip-tilt removed, wavefront errors. It is a 76segment adaptive mirror with 10 segments across its maximum dimensions, and was manufactured by Trex Enterprises (California, USA). The WHT pupil, as imaged at the mirror, covers only 7.3 of the 10 segments. The additional segments provide for a possible future upgrade to a turbulent-layer conjugation capability. Each segment has dimensions of $7.6 \mathrm{~mm}^{2}$ and the surfaces are aluminium coated. Three actuator sectors are used to move each segment thus providing tip-tilt and piston control. The stroke of each actuator is $6 \mu \mathrm{m}$. Strain gauges are provided to measure and correct the hysteresis present in the actuators. Strain gauge amplifiers are mounted close to the DM (see figure 5). A figure sensing interferometer (not shown in figure 1) is permanently deployed to provide an optical view of the DM.
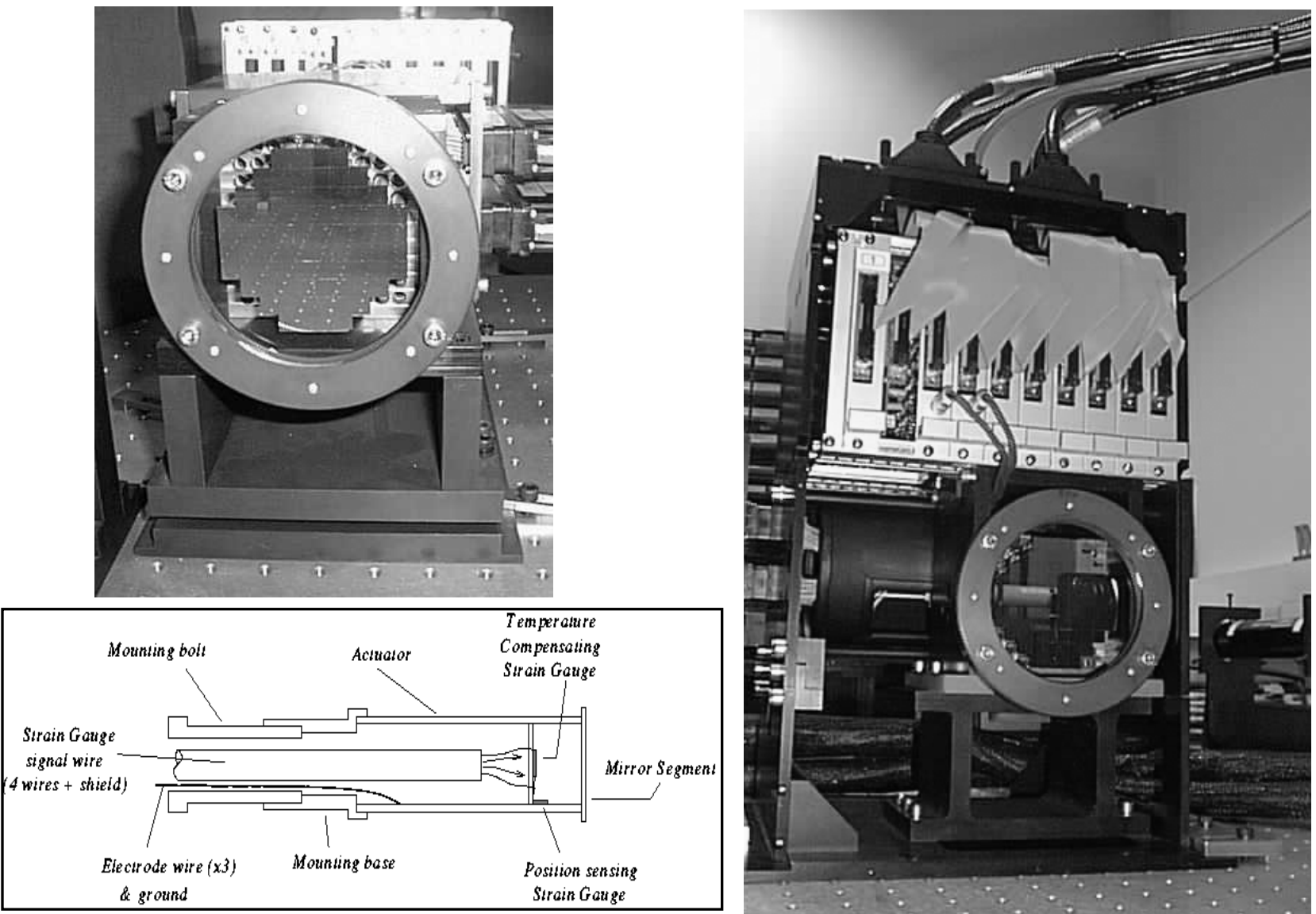

Fig.5: The DM showing its mirror segments (top), a cross-section of an actuator (bottom) and the DM in its mount.

Because the dynamic response and accuracy of the DM depend on the strain gauge feedback control system, those aspects are discussed in the next section.

\section{THE REAL TIME CONTROL SYSTEM (RTCS).}

The NAOMI Real-Time Control System (RTCS) is responsible for processing WFS data to derive positional demand signals for the DM. The RTCS also controls the DM figure using strain-gauge positional feedback. Commands may be sent to the RTCS from remote processes and status information received in reply. It is possible to manipulate control parameters whilst the system is active (i.e. the control loops are closed) and to synchronize updates of many parameters across several processors. The RTCS sends comprehensive diagnostic information streams to remote processes without 
interfering with real-time data flow. These diagnostics are buffered on an independent processor within the RTCS, thus allowing a "history" of control activity to be examined on encountering some particular condition.

Fig.6: The general architecture of the DSP interconnections is illustrated.

\section{Ethernet}

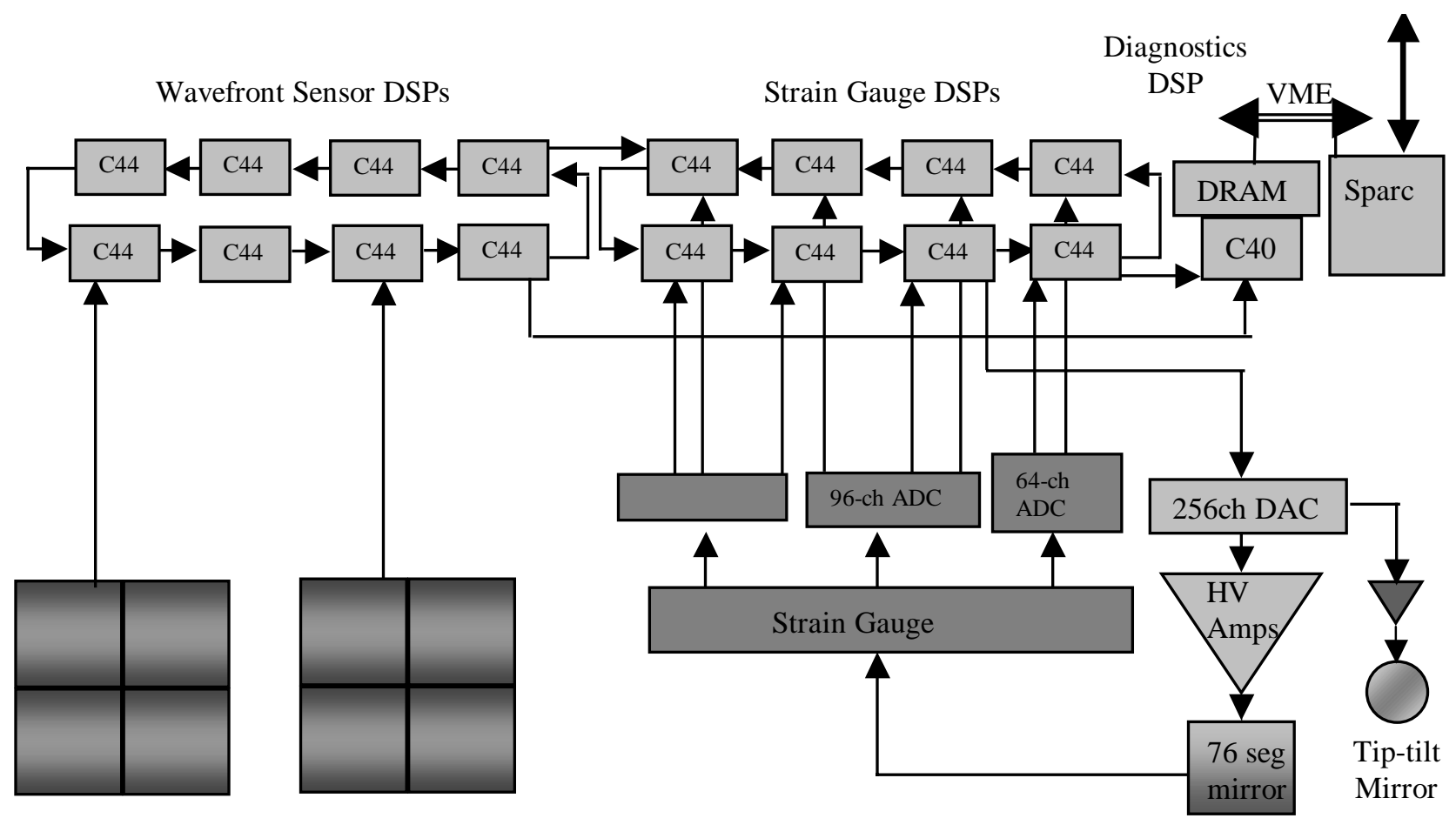

Wavefront Sensor

CCDs

The RTCS is contained in a VME rack that is independent of the WFS and mechanism controllers. Real-time processing is performed on two groups of eight TI TMS320C44 (C44) DSPs, one for WFS processing and one for DM strain gauge feedback. The $\mathrm{C} 44$ has four processor-to-processor bi-directional communication ports, each serviced by an independent Direct Memory Access (DMA) engine. Data can therefore be moved on the communications ports (at up to $20 \mathrm{MBs}^{-1}$ ) without programme intervention. These ports are used to carry incoming data from the WFS and strain gauges and outgoing data to the DM, as well as for real-time interprocessor communication. The two groups of eight processors occupy one VME card each (Blue Wave Systems, UK, DBV44) and have communications ports routed to the panel front, the VME connector and, via Link Interface Adapters (LIAs), to registers mapped to the VME bus. The interprocessor communications ports on each card are connected internally in a ring topology so there is one ring of eight processors for WFS processing and another for strain gauge feedback control. Front panel communications ports carry outgoing diagnostic data from one CPU on each ring to the diagnostic CPU, which occupies a separate VME card. WFS and Strain Gauge data enter on P2 and front-panel communications ports respectively so the VME interfaces only carry traffic to and from the LIA ports, which are used for incoming commands and outgoing status replies. One front panel communications port also links the two rings.

The diagnostic CPU is a TMS320C40 (C40), which has six communications ports instead of four but is otherwise very similar to the C44. It occupies a VME card (a Blue Wave Systems, UK, DBV46) that has panel front communications port connections similar to the DMV44s but also provides a master/slave VME interface to on-board dual-ported memory shared between the C40 and VME interface. This interface is used for retrieving data from the diagnostics buffer held in shared memory and is responsible for the majority of VME bus traffic. 
The controller slot of the VME rack contains the host processor card which is a Force 5V SPARC CPU running the Sun Solaris operating system. This host is an autonomous computer with its own disk and can communicate both via the VME bus and via Ethernet. Its purpose is to mediate between the remote control and display processes and the DSPs.

The two WFS camera interface cards are mounted in the VME rack but do not use the VME bus. They buffer incoming data from each camera and transmit it to $\mathrm{C} 44$ processors via communication ports. Before transmission to the $\mathrm{C} 44 \mathrm{~s}$ is permitted, a frame request from one of the $\mathrm{C} 44 \mathrm{~s}$ must arrive. The interfaces then wait for a Start of Frame word from the cameras before beginning transmission of data to the $\mathrm{C} 44 \mathrm{~s}$. This arrangement allows the $\mathrm{C} 44$ communications port interrupts to remain enabled whilst preventing the $\mathrm{C} 44 \mathrm{~s}$ from being interrupted before they are ready to commence processing. In general this is only required during the transition from unsynchronised to synchronised WFS operation.

The mirror interface card is mounted in the VME rack but does not make use of the VME bus. The mirror demands from the strain gauge DSPs are delivered to the interface using a communications port. A further communications port is used to provide a synchronisation signal.

Time critical WFS signal processing takes place within C44 interrupt service routines, which are triggered by the arrival of the first word of the WFS frame data. Centroiding calculations are adjusted to deal with the different windowing and binning operations, and, as this is based on per-frame header information from the CCD controllers, changes of mode can be made freely by simply commanding the controllers. The only change which cannot be made completely dynamically is changing from an unsynchronised to synchronised mode. This requires interrupts to stop during the transition where unsynchronised frames are arriving from both processors. The WFS processors can currently cope with WFS data rates up to $\sim 500 \mathrm{~Hz}$, although further speed enhancements are planned.

The operation of the strain gauge feedback processing ring is very similar to that of the WFS ring, except that the processing timescales are substantially shorter in order to ensure stable closed-loop operation. The strain gauge ring is currently operated at $3.3 \mathrm{kHz}$ update rate but speed enhancements by a factor $\sim 2$ are planned. The effect of the strain gauge feedback loop is to make the DM appear to the WFS loop to have a linear response. From interferometric measurement, $<\lambda / 150$ non-linearity across $4 \mu \mathrm{m}$ optical path difference has been achieved (at $\lambda=633 \mathrm{~nm}$ ). The strain gauge signals, which provide the WFS feedback, are digitized by 16-bit analogue to digital convertors mounted on VME cards (Pentland, UK). The signals travel through C40 communications ports rather than the VME bus, again to reduce VME bus traffic and avoid contention. The rms measurement noise corresponds to less than 12nm optical path difference on the digitised signal, in the telescope environment (ie $<\lambda / 50$ at $\lambda=633 \mathrm{~nm}$ ).

Automated systems other than the real-time controller use the Experimental Physics Instrument Control Software (EPICS) running on separate Motorola MC68040 processors. One processor deals with WFS control (e.g. selecting the WFS readout mode and an integration time) whilst another coordinates the use of mechanisms. All internal details of the sub-assembly control, such as coordinating the motion of the WFS stages, are dealt with at this level. The WFS and mechanism control processors are hosted in separate VME racks and communicate via Ethernet.

\section{SUPERVISORY SOFTWARE}

A higher level of software runs on workstations (theoretically anywhere with an internet connection) and coordinates the activities of the real-time system and the WFS and mechanism controllers. It also deals with communicating with systems external to NAOMI such as the telescope control system and the science instrument. This degree of coordination is necessary in order to perform observational operations such as dithering and calibration operations such as the removal of non-common path errors. The coordination is performed via a central sequencer, which is responsible for starting and monitoring all system operations (potentially many in parallel) and a process database which contains the system-wide variables. These process variables include a number of mutual exclusion locks to prevent contention between parallel activities.

A unified engineering graphical user interface controls and displays all activities throughout the many susbsytems of NAOMI. In its default state, the display is very simple, and is configured to allow efficient observation. In the case of an error, or some other need to perform detailed diagnostics, the interface guides the user through a set of more detailed panels, which may be activated on demand. 


\section{PERFORMANCE}

\section{REMOVAL OF NON-COMMON PATH ERRORS}

Detailed performance checks were performed on the various subsystems in order to ascertain that they were behaving within specification and the system was then tested as a whole to ensure that it could be aligned and its operation calibrated as required. One particularly important overall check is the ability to remove non-common path errors from the science instrument images of the calibration source with the control loop both open and closed. This is clearly important as it determines the attainable image quality. It is also a stringent test as it involves the optical quality of the whole system (including the WFS in the closed-loop test), and the ability of the system to communicate with an external instrument and to compensate for its optical characteristics.
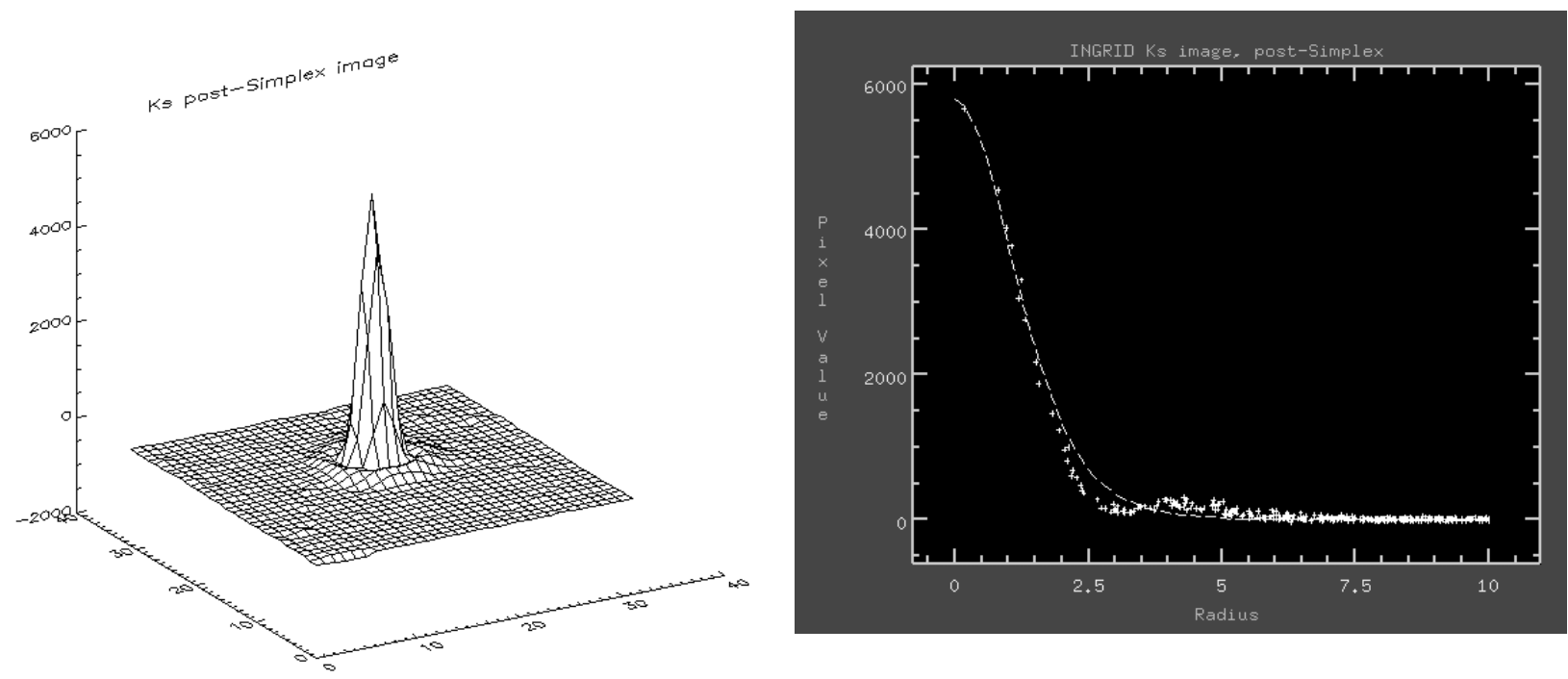

Fig.7: a Ks-band ( 2 micron band) science camera image of the calibration source after removal of non-common path errors. The pixels are equivalent to 0.037 arcsec on-sky. The right hand panel is a radial plot of pixel values.

Figure 7 shows a typical point spread function from the calibration source after removal of non-common-path errors. The final image has a Strehl ratio of $>90 \%$ and its core is diffraction limited. Similar images may be obtained down to 1 micron wavelength on this science camera, although under-sampled by the pixel scale. The removal of the noncommon-path errors was accomplished using a variant of the simplex "hill-climbing" technique employing metrics computed from the science camera itself to optimize the base figure of the $\mathrm{DM}^{4}$.

\section{ON-SKY PERFORMANCE}

Detailed characterisation of NAOMI performance on-sky is currently underway. Preliminary results show that the system is close to its projected performance. Two commissioning images, both of planetary nebulae, are reproduced below. In both cases the central star was used for wavefront sensing. Figure 8 shows an image in the K-band (around 2 microns) where a diffraction-limited core has been obtained. The second image is in a shorter-wavelength narrow band at 1.2 microns. 


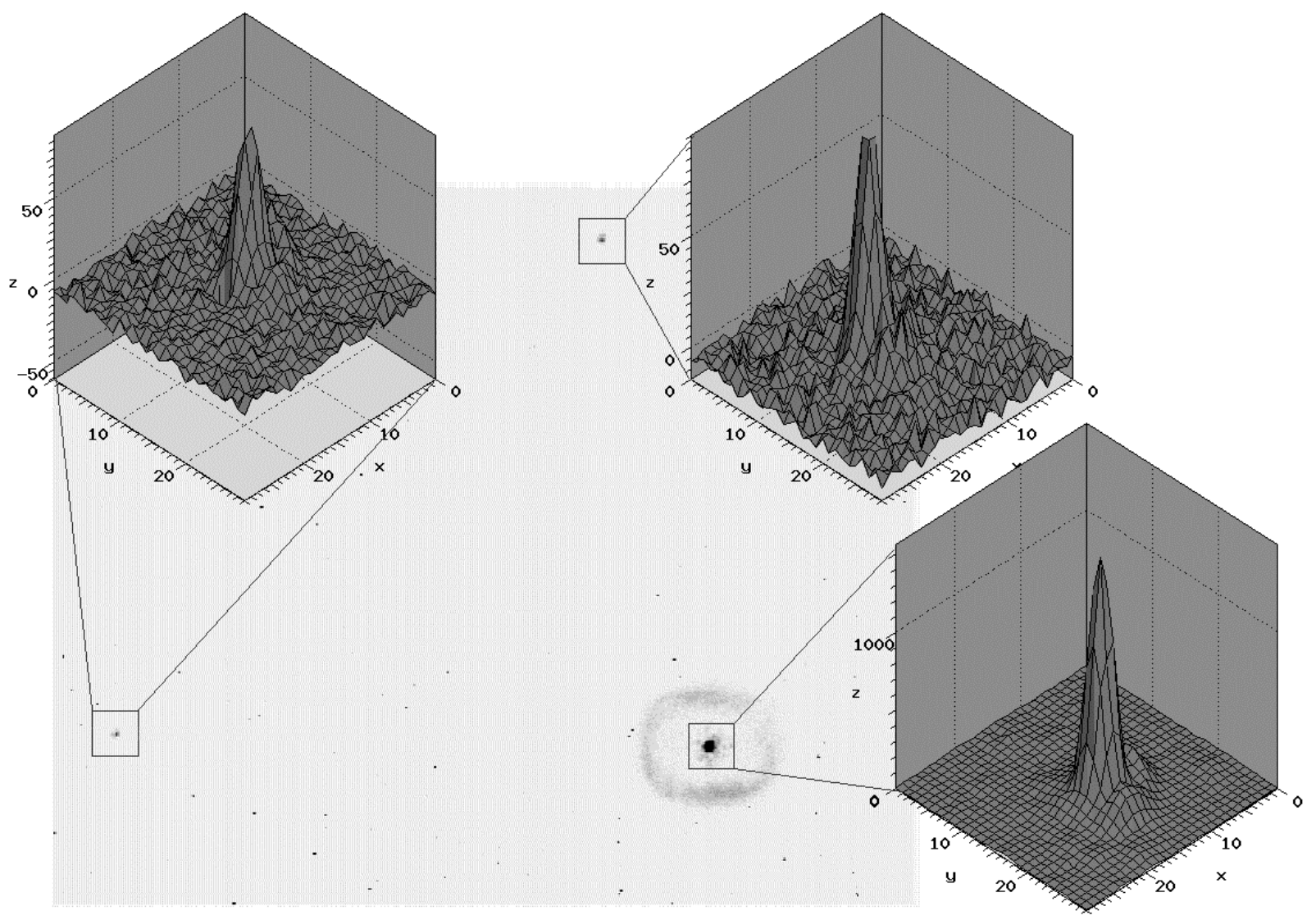

Fig. 8: 90-sec K-band image of planetary nebula BD +30 3369 (nebula diameter 3.8 arcsec top to bottom). The FWHM of the central object is 0.13 ", which is the diffraction limit for this telescope at this wavelength. Note that stars up to 30 arcseconds away from the central object (the guide star) still enjoy good correction. 


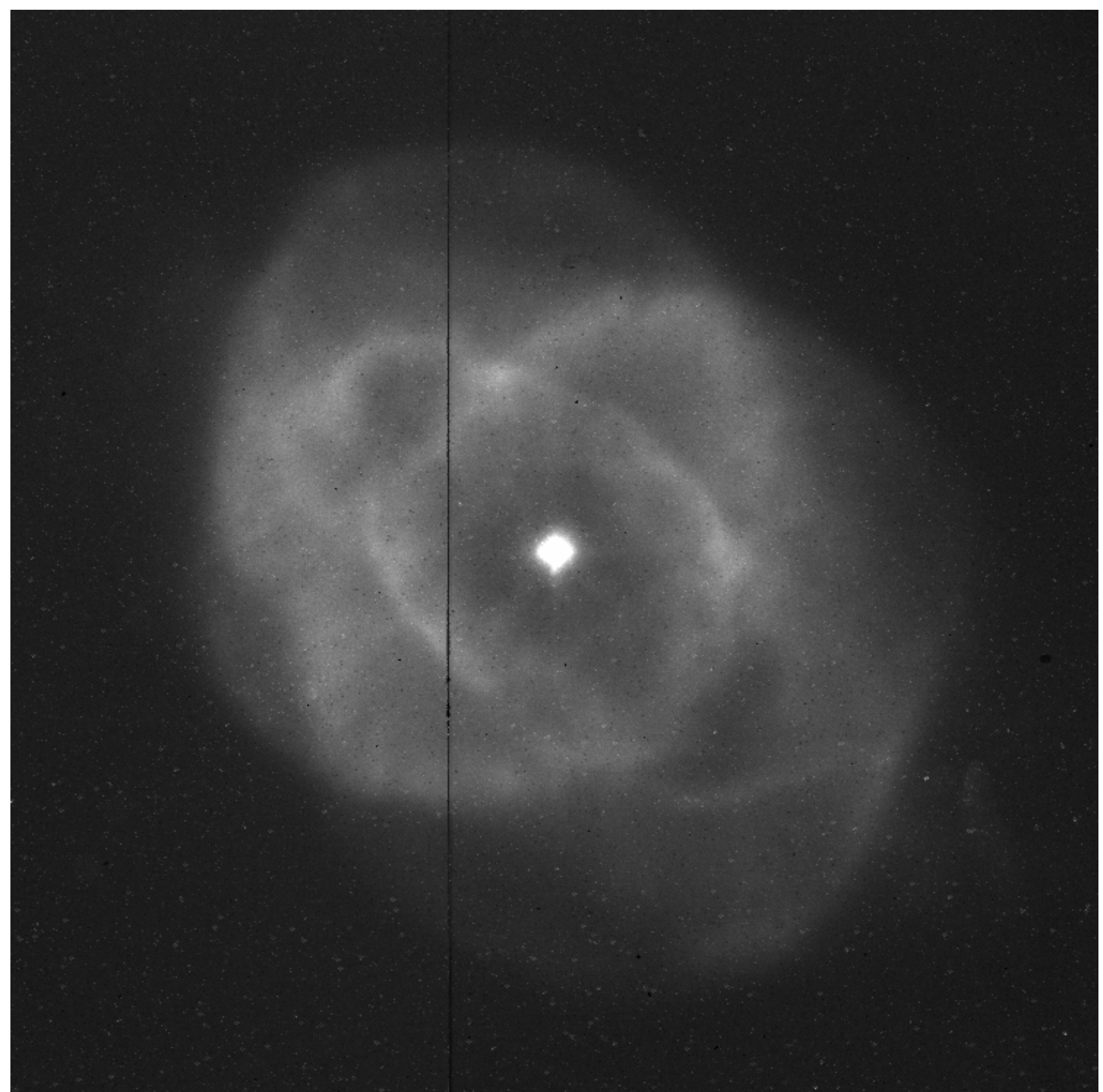

Fig. 9 N6543 planetary nebula in Paschen beta (1.2 microns). 600-sec integration, using central star $(\mathrm{V}=11)$ as a wavefront reference. AO correction from 0.7 to 0.2 arcsec. The finest structures visible in the nebula are $\sim 0.3$ arcsec across. Image is 19 arcsec top to bottom. May 2002.

\section{UPGRADES}

Since its initial configuration, NAOMI has already had one upgrade successfully commissioned: the optical/IR AO coronograph OSCA. OSCA is dealt with in detail in a separate paper by Thompson et al. within these proceedings. Another major upgrade is currently being proposed in the form of a Rayleigh Laser Guide Star for NAOMI. Rutten et al. describe this proposal in a separate paper within these proceedings. This paper also describes the future use of the 
OASIS optical spectrograph with NAOMI, something that has required a number of substantial upgrades, which are now in progress. The principal requirements are for (i) a science dichroic beamsplittter interchange mechanism to replace the single fixed dichroic, and (ii) a completely new Nasmyth mount and enclosure. The first is required in order to feed selected parts of the optical spectrum to OASIS whilst reserving the remainder for wavefront sensing. The second is required to allow NAOMI, INGRID and OASIS to be mounted together.

\section{INSTRUMENTATION}

The instrumentation most commonly used with NAOMI is the WHT's $1024 \times 1024$ pixel HgCdTe near-IR camera INGRID. This is shortly to be augmented by the arrival of the AO-optimised optical integral field spectrograph, OASIS. The use of OASIS with NAOMI is mentioned above and dealt with in more detail in the separate paper by Rutten et al..

\section{ACKNOWLEDGEMENTS}

Numerous other staff members of the Isaac Newton Group of Telescopes, the UK Astronomy Technology Centre at the Royal Observatory Edinburgh, and the University of Durham Astronomical Instrumentation Group made invaluable contributions to the success of the project and have the sincere thanks and gratitude of the authors.

NAOMI is funded by the UK Particle Physics and Astronomy Research Council. The Netherlands N.W.O. is funding the transfer of the OASIS spectrograph which was built by the Observatoire de Lyon, France.

\section{REFERENCES}

1. A. Zadrozny, M. P. J. L. Chang, D. F. Buscher, R. M. Myers, A. P. Doel, C. N. Dunlop, R. M. Sharples, R. L. Arnold, "First Atmospheric compensation with a linearized high-order adaptive mirror-ELECTRA", ESO/OSA Topical Meeting on Astronomy with Adaptive Optics, Ed. D. Bonaccini, ESO Conf. Workshop Proc., 56, pp. 459 - 468, 1999.

2. http://aig-www.dur.ac.uk/fix/projects/naomi/tlsord_files/sciopreq.htm

3. H.T. Barclay, P.H. Malyak, W.H. McGonagle, R.K. Reich, G.S. Rowe, J.C. Twichell, "The SWAT Wavefront Sensor", Lincoln Laboratory Journal, Vol. 5, No. 1, pp. 115, 1992.

4. N. Doble, G. D. Love, D. F. Buscher, R. M. Myers, and Al. Purvis, "The Use of Image Quality Metrics for Correction of Non-Common Path Errors in the ELECTRA Adaptive Optics System”, Proc. Soc. Photo. Opt. Instrum. Eng. 3749 , pp. 785 , 1999. 